Z Gerontol Geriat 2013 · 46:612-612

DOI 10.1007/s00391-013-0528-2

Published online: 20 July 2013

c) Springer-Verlag Berlin Heidelberg 2013
A. Stolzing ${ }^{1} \cdot$ J. Haendeler ${ }^{2,3}$

${ }^{1}$ Translationszentrum für Regenerative Medizin (TRM), Universität Leipzig

${ }^{2}$ Central Institute of Clinical Chemistry and Laboratory Medicine, University of Düsseldorf

${ }^{3}$ Molecular Aging Research, IUF - Leibniz Research Institute for Environmental Medicine, Düsseldorf
The physiological aging process is characterized by progressive structural and functional degenerative changes in many tissues and organs, with a consequent increased incidence of age-related diseases and probability of death [4]. Over the last 30 years it has become evident that genetic aberrations contribute to the onset of diseases and the aging processes. Several gene families and regulatory networks, which modulate the life spans of organisms independent of the environment they live in, have been identified. Thus, several intrinsic pathways that contribute to the aging process are known. However, the role of environmental factors in aging and disorders are still not well understood. There is an obvious correlation between life-style, disease, and aging; in addition, several exogenous factors that have a positive impact on our health status or accelerate aging processes, the onset, and/ or progression of diseases have been identified (e.g., air pollution, caloric restriction, micronutrients, and physical exercise). However, the exact contribution of these extrinsic factors to diseases and aging are largely unknown.

The first lessons for the roles of intrinsic pathways as well as of environmental factors in aging have learned from the roundworm Caenorhabditis elegans ( $C$. elegans), since most genes and environmental interventions that modulate aging processes were first been identified in this system (for review see [5] in this issue). In industrialized nations, cardiovascular disorders are still the major cause of death and aging is one major risk factor for the development of cardiovascular diseases. Structural and functional changes of the major cells, especially endothelial cells, in the cardiovascular systems occur during aging, which are accompanied by an increase in oxidative stress (for review see [2] in this issue). Besides cardiovascular disorders, chronic respiratory diseases also increase with age. Several studies provide evidence that environmental factors enhance pulmonary diseases by inducing intracellular pathways leading to cellular senescence (for review see [1] in this issue). In recent years, it has become clear that not only somatic cells but also adult stem cells undergo age-related changes. Therefore, it is mandatory to understand these processes in adult stem cells. Recent studies demonstrated that micro RNAs are important regulators of gene expression. Micro RNAs seem to co-regulate stem cell properties and could, therefore, be involved in the age-induced changes of these cells (for review see [3] in this issue).

Taken together, we are still at the beginning of understanding aging processes on the molecular level. Therefore, further investigations in model organisms like $C$. elegans, mice as well as in human cells and tissues are needed, as it is of fundamental importance to investigate the molecular bases underlying the physiology as well as the pathophysiology of aging processes in order to develop preventive interventions aimed at postponing functional decline, thus, prolonging healthy lifespan.

\section{A. Stolzing}

\section{J. Haendeler}

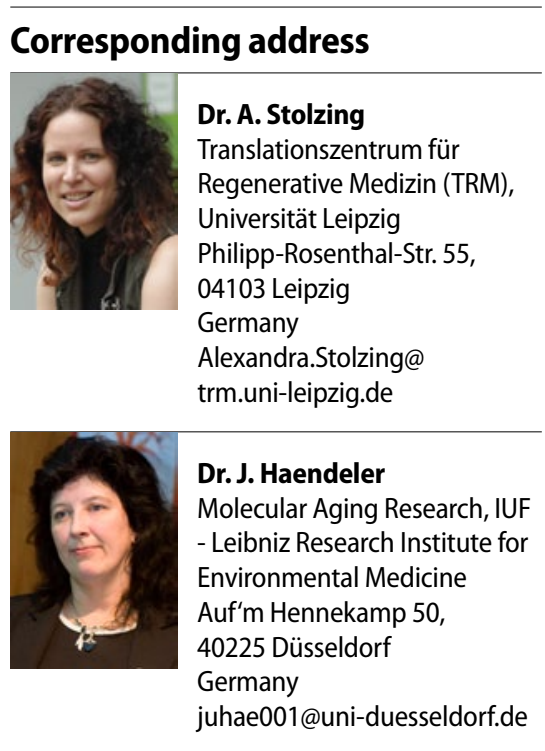

\section{References}

1. Bartling B (2013) Cellular senescence in normal and premature lung aging. $Z$ Gerontol Geriatr

2. Czypiorski P, Rabanter LL, Altschmied J et al. (2013) Redox balance in the aged endothelium. Z Gerontol Geriatr

3. Hodzic M, Naaldjik Y, Stolzing A (2013) Regulation of aging in adult stem cells by microRNA. Z Gerontol Geriatr

4. Kirkwood TB (2008) A systematic look at an old problem. Nature 451:644-647

5. Torgovnick A, Schiavi A, Maglioni S et al. (2013) Healthy aging: what can we learn from $C$. elegans? Z Gerontol Geriatr 